\title{
Study of Complex Cathode Emission Characters
}

\author{
Y. Zhang, F. Song And F. Xiang
}

Institute of Applied Electronics, CAEP, P.O. Box 919-1015, Mianyang 621900, China

Based on the study of explosive emission, the dielectric enhanced electric-field emission model is brought forward, and the metal-dielectric complex cathode material is manufactured. Based on the investigation of surface-flashover explosive emission, the graphite-carbon fiber complex cathode is manufactured, too. Then the experiments are developed respectively, and the results show the electron emission density of these two complex cathode materials is also more than $17 \mathrm{kA} / \mathrm{cm}^{2}$, and their lifetime is exceeding $10^{5}$ pulses.

PACS numbers: 52.25.Tx; 52.59.Mv; 52.80.Qj

\section{Introduction}

High-current electron beams $(\geq 100$ A) produced by high-voltage $(\geq 100 \mathrm{kV})$ generators have found widespread use in such diverse applications as high power microwave generation, free electron lasers, pumping of excimer lasers, and material surface modification. In these various devices, the beam electrons are often generated by any process of thermionic cathodes emission, photoelectric emission and electric-field enhanced emission. The intense electron-beam source in generating high power microwaves has beam density and beam current that are very high and the usual emission processes cannot satisfy these requirements other than the explosive electron emission (EEE). The electron-beam density and current provided by the explosive electron emission is as high as $\mathrm{kA} / \mathrm{cm}^{2}$ and MA, respectively $[1,2]$.

For the explosive emission cathodes, the initial electron is emitted from the points that have a large electric-field enhancement coefficient and small potential function. When the high voltage pulse is applied between the cathode and anode, the plasma will appear at these points of cathodes surface, electrons of the plasma will be continuously drawn out and accelerated by the electric field. There are two ignition mechanisms in this physics process, electric-field enhanced explosive emission and surface-flashover explosive emission. This article will introduce these two explosive emission mechanisms and the related initial experimental investigations.

\section{Field-enhanced explosive emission}

Tip enhanced electric field is the primary ignition mechanism of metal and graphite cathodes. Micro-tips exist on all metal surfaces, which is called metal crystal beard usually with $10^{-4} \mathrm{~cm}$ high and $10^{-5} \mathrm{~cm}$ radius [3], and its density is estimated about $10^{4} / \mathrm{cm}^{2}$.

When the high voltage pulse is applied between the cathode and anode, the microscopic $E$-field of the cathode surface will be enhanced a thousand times over the macroscopic $E$-field during the voltage pulse rising. Then the field-enhanced electron emission appears, and the electron emission density increases rapidly. Here the emission is influenced by space charge and converts to the space charge limited regime gradually. During this period, the cathode material atom or molecule explodes and the plasma columns appear because of resistive heating of metal crystal beard, that is cathodes bright spot, see Fig. 1. Because the cathodes bright spots rapidly hydrodynamically expand and incorporate, the cathodes plasma shell is formed rapidly. Then the effective emission area increases sharply, and the intense e-beam is produced. That is the typical micro-point enhanced electric-field explosive emission. In this course the plasma temperature is as high as several thousands $\mathrm{K}$, so the expanding speed is quickly $2 \mathrm{~cm} / \mu \mathrm{s} \approx 6 \mathrm{~cm} / \mu \mathrm{s}$, and the diode $\mathrm{A}-\mathrm{K}$ gap will be closed in short time [4-8].

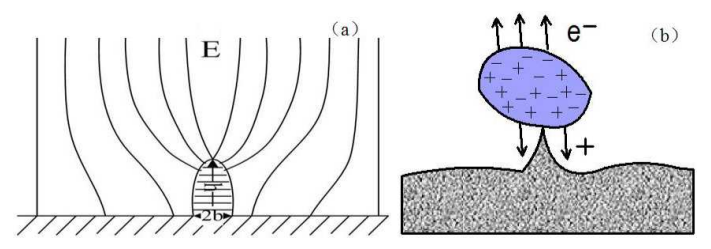

Fig. 1. Micro-point enhanced electric-field (a) and sharp-angled explosive emission (b).

In fact, the presence of various dielectric inclusions and absorbed gas of cathode surface are the primary factors in producing the initial plasma spots, and then the second emission is beginning because of the resistive heating. More and more dielectric inclusions and absorbed gas as well as the steam of cathode materials are continually released from the cathode along with the temperature rise. Then the bright spots explode and the local plasma are produced, while the local plasma rapidly hydrodynamically expands and incorporates, the cathode plasma emission area is formed rapidly. That is the typical dielectric enhanced electric-field explosive emission, such as Fig. 2 shows.

Therefore, we can see that for the initial stage the emission properties of the cathodes are mainly determined by the initial state of the surface layer, and the cathode plasma results from the ionization of various dielectric inclusions and absorbed gas of cathode surface.

Based on the study of dielectric enhanced electric-field explosive emission, the dielectric enhanced electric- 


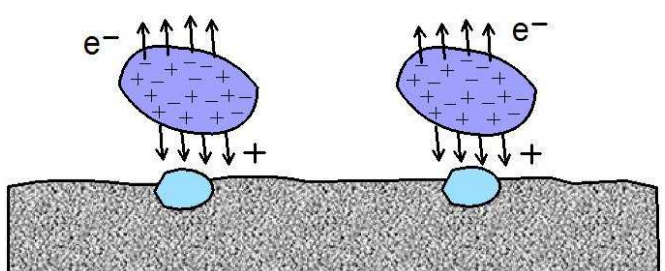

Fig. 2. Dielectric enhancement electric-field emission model.

-field emission model is brought forward, and the metaldielectric complex cathode material is manufactured. This complex cathode is made of metal (copper) and high dielectric particulate (ceramic) which are mixed and pressed, the dielectric particulates are the enhanced electric-field points, and the dielectric constant is higher, the enhanced electric-field coefficient is higher.

\section{Surface-flashover explosive emission}

The physical mechanisms involved in the explosive emission process for dielectric cathode materials such as velvet were investigated. We have determined that explosive emission from velvet is initiated by a surface flashover mechanism. The surface discharge gives rise to a cold dense plasma/gas column. A phenomenological model based on resistive heating of the plasma columns appears to give a good estimate for the final diode-plasma closure velocity.

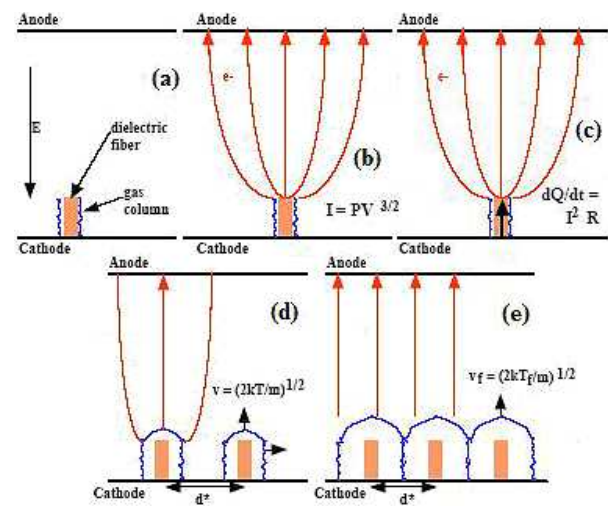

Fig. 3. Surface-flash explosive emission [10]: (a) Surface flashover generates a cold, dense plasma/gas column. (b) The applied electric field extracts a spacecharge-limited current flow. (c) The flow of current resistively heats the gas column. (d) The gas columns expand at a rate determined by the gas temperature. (e) The gas continues to expand into the anode-cathode gap.

Miller has investigated and experimented the physical mechanisms of dielectric cathode materials such as velvet $[9,10]$. He considered that surface flashover explosive emission was the main ignition mechanism in emitting electrons. Usually, there are five steps involved in the explosive emission process for dielectric fibers, as Fig. 3 illustrates.

The temperature of plasma which produced by surface flashover explosive emission is lower than that produced by field-enhanced explosive emission, so the expand speed is about $0.2 \mathrm{~cm} / \mu \mathrm{s} \approx 2 \mathrm{~cm} / \mu \mathrm{s}$.

Surface flashover explosive emission is the main mechanism for carbon fiber, and it has low emission threshold, intense current density and high sublimation temperature, so carbon fiber can be used for the long lifetime cathode. We use carbon fiber as the frame and fill in graphite particulate, then the graphite-carbon fiber complex cathode is manufactured.

\section{Experiments of complex cathode emission}

Based on the study of dielectric enhanced electric-field emission model the metal-dielectric complex cathode is manufactured. Based on the investigation of surface flashover explosive emission, the graphite-carbon fiber complex cathode is manufactured, too. Figures 4

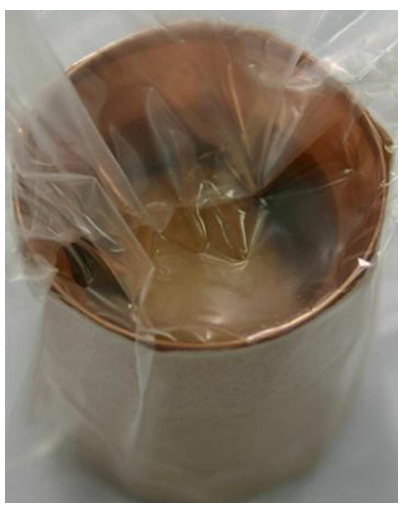

Fig. 4. Copper-ceramic complex cathode.

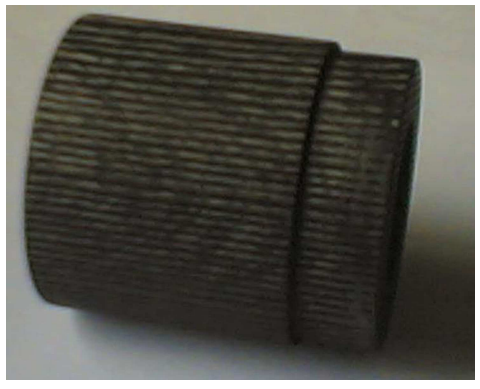

Fig. 5. Graphite-carbon fiber complex cathode.

and 5 present a photo of metal-dielectric complex cathode and graphite-carbon fiber complex cathode, respectively. Then the experiments are performed on a SINUS700 accelerator with 100 pps repetition rate.

Measurements were performed with the use of a Lecroy Waverunner digital oscilloscope $(1 \mathrm{GHz}, 5 \mathrm{GS} / \mathrm{s})$. Waveforms of vacuum diode voltage and beam current were 
monitored. The diode voltage was measured using a capacitive voltage divider, the current measurements were performed with a Rogowski coil. The emissive properties of the cathodes were estimated from the pulse peak current and the time of current rise to a level of $12 \mathrm{kA}$.

Figure 6 presents 100 overlap waveforms of copperceramic complex cathode after $10^{4}$ pulses. Figure 7 presents 100 overlap waveforms of graphite-carbon fiber complex cathode after $10^{4}$ pulses.

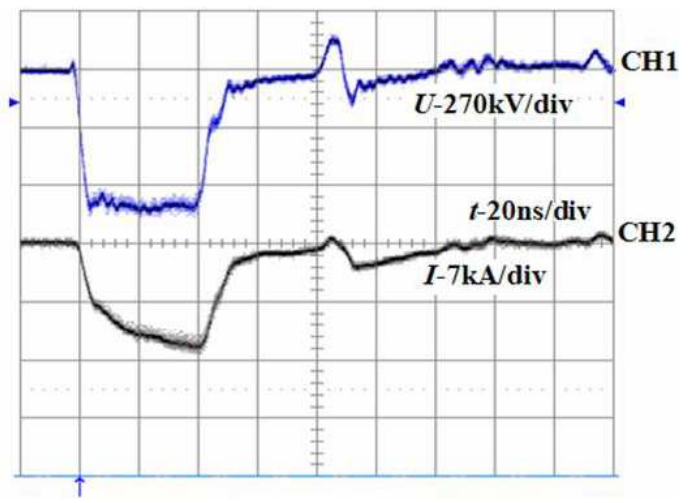

Fig. 6. Waveforms of copper-ceramic cathode.

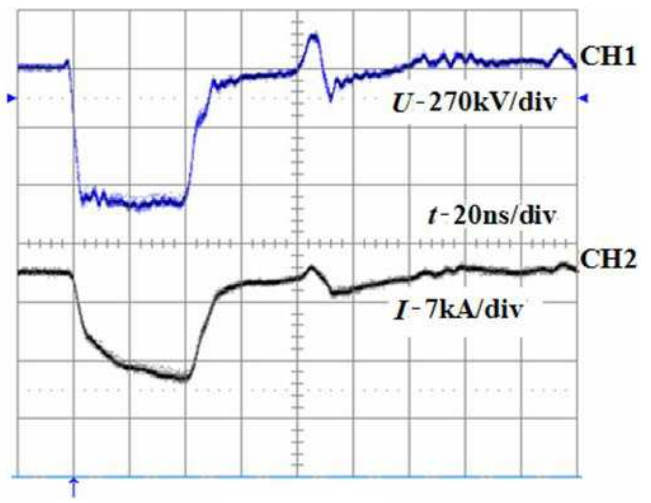

Fig. 7. Waveforms of graphite-carbon fiber cathode.

As mentioned above, these two complex cathodes demonstrated their predominant emissive properties as the number of pulses were increased. In particular, an increase in current rise-time was observed, attesting to a reduced distribution density of emission centers. In the figures, we can see the diode voltage $(\mathrm{CH} 1)$ is $600 \mathrm{kV}$, and the current $(\mathrm{CH} 2)$ is $12 \mathrm{kA}$, the pulse duration is about $40 \mathrm{~ns}$, and the e-beam emission density is as high as $17 \mathrm{kA} / \mathrm{cm}^{2}$. We also can see there are no obvious changes in emission properties of these two complex cathodes after $10^{4}$ pulses, so their lifetime is estimated exceeding $10^{5}$ pulses.

Our experiment results agree well with the conclusion of Gunin [11]. According to their investigation results, the lifetime of pyrolytic graphite and metal- dielectric complex cathode are more than $10^{6}$ pulses with $10^{4} \mathrm{~A} / \mathrm{cm}^{2}$ current density.

\section{Conclusion}

According to the experimental results, the theoretic analysis mentioned above and the investigation results of Gunin, we can see that pyrolytic graphite cathode is the typical micro-point enhanced electric-field explosive emission, copper-ceramic complex cathode is the typical dielectric enhanced electric-field explosive emission, and graphite-carbon fiber complex cathode is the typical surface-flashover explosive emission. Pyrolytic graphite is made of carbon granules, the micro-points above the surface are the $E$-field enhanced points, and then result in explosive emission. For the metal-dielectric complex cathode, the dielectric particulates in the metal are the $E$-field enhanced points, and the dielectric constant is higher, the enhanced electric-field coefficient is higher. Because the dielectric particulates are uniformly distributed in the metal, even when the cathode surface is ablated, but the inner compositive materials are same as its surface, the emissive properties have not obvious changes, which is not as pure metal cathode, so the metal-dielectric complex cathode has long lifetime. Graphite-carbon fiber complex cathode combines the excellences of pyrolytic graphite and carbon fiber, it has not only high melting point, but also low emission threshold and low plasma expanded speed, so it can be used for the long lifetime cathode.

\section{References}

[1] G.A. Mesyats, IEEE Trans. Plasma Sci. 19, 683 (1981).

[2] A.V. Gunin, A.I. Klimov, S.D. Korovin, I.K. Kurkan, I.V. Pegel, S.D. Polevin, A.M. Roitman, V.V. Rostov, A.S. Stepchenko, E.M. Totmeninov, IEEE Trans. Plasma Sci. 1, 141 (1997).

[3] R.B. Miller, An Introduction to the Physics of Intense Charged Particle Beams, Plenum Press, New York 1982.

[4] S.D. Korovin, E.A. Litvinov, G.A. Mesyats, A. Murzakaev, V. Rostov, V. Shpak, S. Shunailov, M. Yalandin, Techn. Phys. Lett. 30, 813 (2004).

[5] S.D. Korovin, G.A. Mesyats, I.V. Pegel, S.D. Polevin, V.P. Tarakanov, IEEE Trans. Plasma Sci. 28, 485 (2000).

6] G.Z. Lin, J. Sun, H. Shao, et al., High Power Laser Particle Beams 17, 249 (2005).

[7] G.Z. Liu, J. Sun, H. Shao, Z.F. Yang, Shiyan Yu Yanjiu 27, 1 (2004).

[8] A.V. Gunin, V.F. Landl, S.D. Korovin, IEEE Trans. Plasma Sci. 28, 537 (2000).

[9] R.B. Miller, Proc. SPIE, 3158, 2 (1997).

[10] R.B. Miller, J. Appl. Phys. 84, 739 (1998).

[11] A.V. Gunin, V.F. Landl, S.D. Korovin, G.A. Mesyats, V.V. Rostov, Techn. Phys. Lett. 25, 922 (1999). 